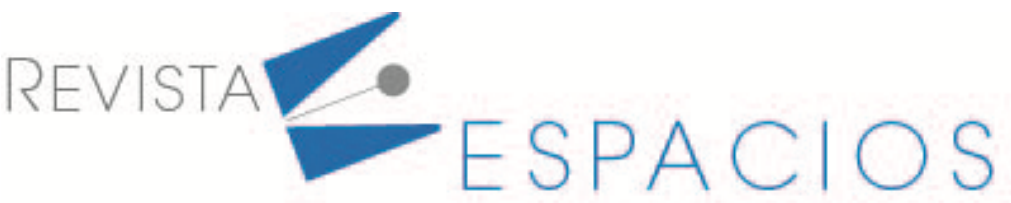

\section{Caminhos para smart grids: análise global do ponto de vista científico e intelectual}

\section{Paths to smart grids: global analysis from the scientific and intellectual standpoint}

\author{
DE ATAIDE, Lucas $^{1}$ \\ NEUENFELDT JÚNIOR, Alvaro ${ }^{2}$ \\ SILUK, Julio ${ }^{3}$ \\ MICHELIN, Cláudia ${ }^{4}$ \\ FRANCESCATTO, Matheus ${ }^{5}$
}

\begin{abstract}
Resumo
A geração de energia elétrica de maneira centralizada, até hoje ainda majoritária em alguns países, enfrenta uma concorrente a altura: a geração descentralizada, ou geração distribuída, que preza pelas energias renováveis alternativas e redes inteligentes (smart grids). O objetivo deste artigo é apresentar um recorte temporal das atividades científicas e intelectuais/industriais mundiais mais significativas relacionadas a smart grids. Espera-se do relacionamento entre as perspectivas de análises que auxiliem pesquisadores e stakeholders da cadeia energética na identificação de tendências de possíveis cenários envolvendo determinado conceito ou tecnologia em torno de smart grids.

Palavras-chave: prospecção tecnológica; redes inteligentes; propriedade intelectual; energia.
\end{abstract}

\begin{abstract}
The centralized generation of electricity, initially and until today majority in some countries, faces today a same level competitor: decentralized generation, or distributed generation, focused on alternative renewable energy and smart grids. The aim of this study is to present a temporary clipping of the most significant scientific and intellectual/industrial activity related to smart grids. It's looked forward that the relation between these two perspectives of analysis helps researchers and stakeholders of the energy chain to identify tendencies of possible scenarios involving concepts or technologies regarding smart grids.
\end{abstract}

Keywords: technological prospecting; smart grids; intellectual property; energy.

\footnotetext{
${ }^{1}$ Departamento de Engenharia de Produção e Sistemas. Universidade Federal de Santa Maria. lucas.moreira@ecomp.ufsm.br

2 Departamento de Engenharia de Produção e Sistemas. Universidade Federal de Santa Maria. alvaroj.eng@gmail.com

3 Departamento de Engenharia de Produção e Sistemas. Universidade Federal de Santa Maria. jsiluk@ufsm.br

${ }^{4}$ Departamento de Ciências Contábeis. Universidade Federal de Santa Maria. claudia.michelin@ufsm.com

${ }^{5}$ Departamento de Engenharia de Produção e Sistemas. Universidade Federal de Santa Maria. matheusfrancescatto@hotmail.com
} 


\section{Introdução}

Os fenômenos naturais causados pelas energias solar e gravitacional sempre instigaram o ser humano a captar seus efeitos e utilizá-los em seu benefício. Por exemplo, o calor e os ciclos hidrológicos foram e vão continuar sendo os principais meios que permitem a geração de energia elétrica, um fator chave para o desenvolvimento econômico e social das nações.

Em função do aumento populacional e dos adventos tecnológicos, as pessoas necessitam de mais energia para criar um ambiente de vida melhor. $O$ aumento no consumo de energia requere acionamento de mecanismos de compensação energética - fontes que se utilizam da queima de combustíveis fósseis e causam uma série de problemas ambientais, como mudanças climáticas, poluição e aquecimento global e impactam a vida em todas as suas formas (Sampaio; González, 2017).

Durante o estudo e o desenvolvimento de novas tecnologias, encontram-se etapas importantes para pesquisadores, empresas e demais stakeholders. Algumas delas são os processos de registros de novas pesquisas e tecnologias, certificados por sistemas e bases de dados unificadas globalmente. Após o registro, o acesso ao conhecimento passou a se apresentar de uma forma facilitada e, para frutificar ainda mais esses mecanismos, pode-se utilizar de métodos baseados no monitoramento da evolução temporal, um acompanhamento sistemático e contínuo dos fatos, identificando fatores que provocam mudanças na sua evolução (Caruso; Tigre, 2004; Falbo et al., 2015). No presente estudo há o uso dessa lógica com um enfoque voltado para a área de Smart Grids (SG), ou Redes Elétricas Inteligentes. SG buscam uma nova forma de distribuição de energia renovável, controlável remotamente e automatizada.

O setor elétrico brasileiro enfrentou grave crise energética entre 2013 e 2015, expondo a fragilidade do sistema de geração do país e despertando a preocupação em descentralizar e diversificar a sua matriz elétrica (Silva; Vieira, 2016). Nessa esfera, a geração distribuída encontra caminhos para difusão no Brasil, seguida por um desconhecimento dos efeitos, tanto técnicos quanto ambientais, da utilização deste recurso no Sistema Elétrico Brasileiro (Carvalho; Delgado, 2017).

Os principais incentivos no Brasil são as chamadas públicas da Agência Nacional de Energia Elétrica (ANEEL) voltadas para operação de plantas fotovoltaicas no país, a isenção do Imposto sobre Produtos Industrializados para energia elétrica, a isenção do Imposto sobre Circulação de Mercadorias para equipamentos e componentes para aproveitamento da energia solar e a redução do Imposto de Importação destinado à produção de equipamentos de geração fotovoltaica (Rosa et al., 2016; Rediske et al., 2018; Garlet et al., 2019). Ademais, há o apoio do Banco Nacional de Desenvolvimento Econômico e Social (BNDES) para financiar, com taxas diferenciadas, projetos de geração distribuída em hospitais e escolas públicas, e ainda o Plano Inova Energia, do BNDES, da ANEEL e da Financiadora de Estudos e Projetos (FINEP) para estimular a pesquisa e inovação em energias alternativas, como a solar (Ministério de Minas e Energia, 2016).

Todo aparato regulatório brasileiro ainda é incipiente, análogo ao nível de atividade no setor de geração distribuída e no uso de fontes alternativas, como a solar e eólica, em comparação com as oportunidades que dispõe. Atrelado a isso, as interconexões entre tecnologias e sistemas socioeconômicos e organizacionais estão se tornando mais amplas e profundas, abrindo espaços para a fase de pesquisa, dado sua importância particular para gerar novas hipóteses, conhecimentos, conceitos e inovações.

Considerando as fragilidades geradas pela falta de diversidade na matriz de geração elétrica brasileira e os benefícios verificados provenientes da expansão da geração distribuída, esta pesquisa discute a seguinte problemática: "Como auxiliar no direcionamento da atenção de pesquisadores para melhorar a compreensão e disseminação sobre a geração distribuída nos próximos anos?". 
O objetivo principal deste trabalho é apresentar um recorte temporal das atividades científicas e intelectuais/industriais (patentes) mundiais mais significativas, apresentando relação com os indicadores brasileiros. Espera-se do relacionamento entre essas duas perspectivas de análises que auxiliem demais pesquisadores na identificação de tendências de possíveis cenários envolvendo determinado conceito ou tecnologia em torno de SG.

Esse artigo está organizado em cinco seções. A Seção 1 corresponde a introdução, apresentando o problema e o objetivo principal da pesquisa. A Seção 2 descreve o referencial teórico sobre smart grids e prospecção tecnológica. Na Seção 3 estão descritos os passos metodológicos, enquanto a Seção 4 apresenta os resultados obtidos e a análise realizada. Finalmente, a Seção 5 é organizada para retomar as principais conclusões sobre o projeto desenvolvimento.

\section{Referencial teórico}

Com o objetivo de contextualizar o conceito selecionado para análise dessa pesquisa, esta sessão visa fundamentar o termo smart grids. Além disso, prospecção tecnológica também é um conceito apresentado nessa sessão.

\subsection{Smart grids}

Um dos sistemas mais dinâmicos e essenciais para os seres humanos é o abastecimento energético, pelo desenvolvimento econômico e social que possibilitam (Schandl et al., 2016; Cavallo et al., 2018). De fronte a isso, o modelo tradicional de geração de eletricidade baseada em mecanismos hidrológicos, centralizado, depara-se com as redes modernas, que utilizam conceito de smart grids, apresentando inovações para obter maior flexibilidade como, por exemplo, a inserção de unidades geradoras descentralizadas no sistema, configurando a geração distribuída (Dağ et al., 2017).

A Geração Distribuída (GD) significa que a origem de energia elétrica está junto ou próxima dos consumidores, independente da potência, tecnologia ou da fonte de energia. A GD está se tornando cada vez mais importante nos sistemas de energia, descentralizando os mesmos e causando uma transição. As dinâmicas envoltas nesses processos transitórios são difíceis de serem previstas, portanto, representam grande desafio para stakeholders da cadeia energética. Uma análise sistêmica do aspecto tecnológico inovador precisa ser essencial para atingir um entendimento holístico da interação dos diferentes elementos da GD e para criar melhores soluções tecnológicas ainda (Kubli; Ulli-Beer, 2018; Rosa et al., 2016).

Uma rede elétrica inteligente é concebida como uma rede elétrica que pode fornecer eletricidade de maneira inteligente e controlada, de pontos de geração a consumidores ativos. A rede apresenta uma característica flexível conhecida como resposta à demanda, que basicamente promove a interação entre a capacidade de resposta dos clientes com a demanda do sistema. Por isso, SG podem oferecer uma ampla gama de benefícios potenciais na operação e expansão do sistema e na eficiência do mercado. Com essa inteligência, é possível melhorar a confiabilidade do sistema de energia e, a longo prazo, reduzir a demanda de pico, reduzindo os investimentos gerais de custo de fábrica, de capital e adiando a necessidade de atualizações de rede (Siano, 2014).

\subsection{Prospecção Tecnológica}

Países e instituições avançados colocam, cada vez mais, ênfase em processos de estudos do futuro (foresight), que criam espaços de diálogo estruturado com foco em inovações sistêmicas ou transformadoras (CGEE, 2014). O desenvolvimento de inovações depende de inúmeras competências de ordens técnica, organizacional e gerencial, e as organizações que trabalham com PD\&l devem estar avançadas com relação aos seus processos 
estratégicos se quiserem vencer os desafios tecnológicos futuros e gerar vantagens competitivas (EMBRAPII, 2014; Scarano et al., 2014; Neuenfeldt Júnior et al., 2015).

Para as empresas, a missão do planejamento é, além de prestar séria atenção para os desafios atuais e prover abordagens temporárias para tal, pensar sobre os desafios futuros e como prevenir-se a eles (Sadoogi; Najafzadeh, 2016). Nessa perspectiva, para atingir um bom desempenho no delineamento de cenários futuros, é necessário utilizar instrumentos que definam estruturalmente um sistema sob estudo, a fim de construir procedimentos para modelar os seus relacionamentos internos e externos (Battistella, 2014; Costa et al., 2015; Siluk et al., 2017; Júnior et al., 2018; Nascimento et al., 2019).

No Brasil, o Instituto Nacional da Propriedade Industrial (INPI) é o órgão que utiliza documentos de patente como fonte de informação tecnológica e estatística para aperfeiçoar, disseminar e gerir o sistema brasileiro de concessão e garantia de direitos de Propriedade Intelectual (PI) (Mayerhoff, 2009). No âmbito da prospecção tecnológica, podem ser utilizadas metodologias diversas, conforme exemplificado pela Tabela 1.

Tabela 1

Atividades de Prospecção no Brasil.

\begin{tabular}{|c|c|c|c|}
\hline Atividade & Ano & Temas estudados & Métodos utilizados \\
\hline Programa Prospectar & $\begin{array}{l}2000- \\
2003\end{array}$ & $\begin{array}{l}\text { Aeronáutica, agricultura, energia, saúde, } \\
\text { recursos hídricos, materiais, espaço, } \\
\text { telecomunicações e TI }\end{array}$ & Brainstorming, Delphi e priorização \\
\hline $\begin{array}{l}\text { Programa Brasileiro de } \\
\text { Prospecção } \\
\text { Tecnológica (para } \\
\text { 2016) }\end{array}$ & 2002 & $\begin{array}{l}\text { Construção civil, indústria têxtil e de } \\
\text { vestuário, plástico, madeira e mobília }\end{array}$ & $\begin{array}{c}\text { Análise de fatores críticos, análise } \\
\text { de impactos cruzados e de } \\
\text { tecnologias-chave, modelagem e } \\
\text { cenários }\end{array}$ \\
\hline $\begin{array}{l}\text { Prospecção } \\
\text { Tecnológica Setorial e } \\
\text { Regional (CGEE) }\end{array}$ & $\begin{array}{l}2002- \\
2007\end{array}$ & $\begin{array}{l}\text { Materiais avançados, biocombustíveis, } \\
\text { biotecnologia, mudanças climáticas, } \\
\text { energia, recursos hídricos e } \\
\text { nanotecnologia }\end{array}$ & $\begin{array}{l}\text { Brainstorming, Delphi, } \\
\text { questionários, workshops, etc. }\end{array}$ \\
\hline
\end{tabular}

Fonte: Adaptado de Chan e Daim (2012)

Para o Brasil, cujo desenvolvimento socioeconômico ainda é incompleto, um dos desafios é aumentar a competitividade da indústria nacional, o que depende em grande parte da sua capacidade inovadora (Mazzucato; Penna, 2016). Tal premissa depende do aumento dos investimentos empresariais em pesquisa, desenvolvimento e inovação (PD\&l) e do estímulo aos projetos de maior/melhor conteúdo tecnológico - mais arriscados, mas que compensam - pois adicionam barreiras mais robustas contra a concorrência e podem produzir maior impacto econômico (EMBRAPII, 2015). A atividade tecnológica inovativa do Brasil apresenta uma trajetória contínua de distanciamento dos países considerados mais inovadores (Figura 1). 
Figura 1

Índices globais de inovação (2011 - 2018).

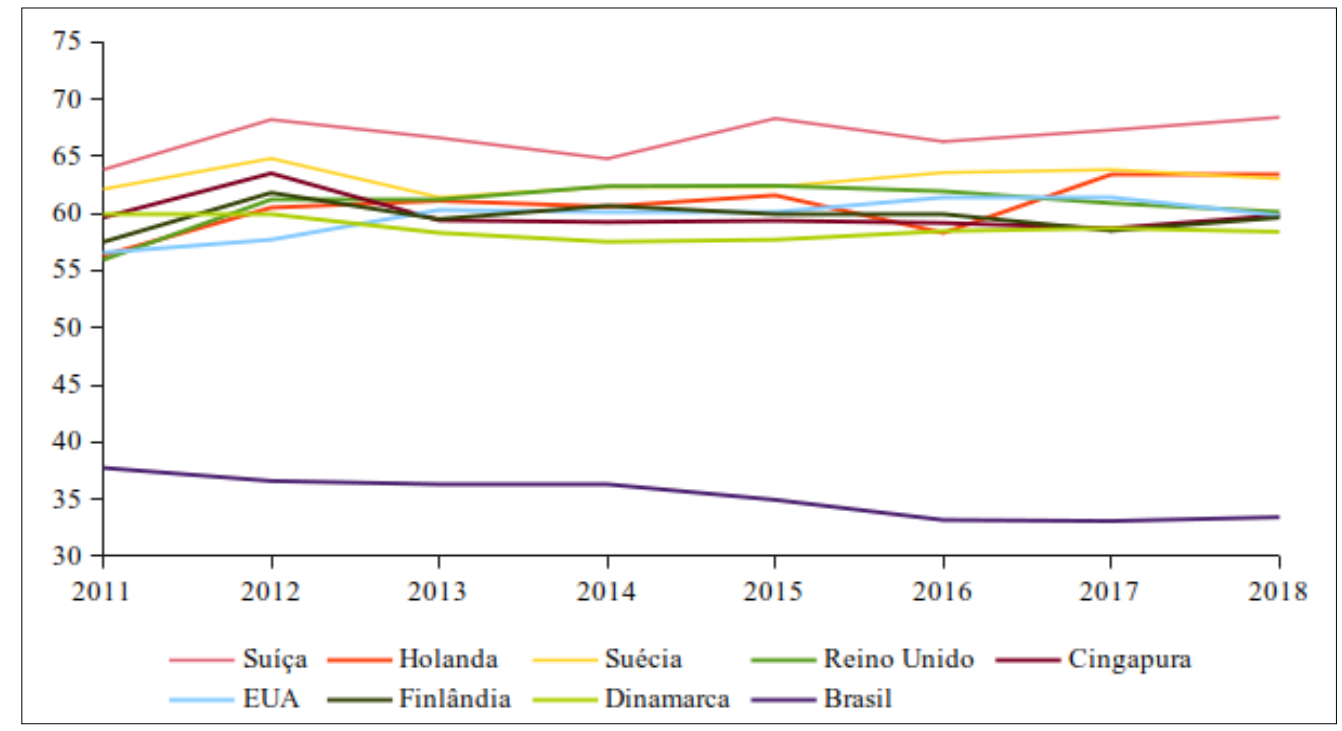

Fonte: WIPO (2011, 2012, 2013, 2014, 2015, 2016, 2017, 2018).

Uma das causas para o Brasil não ter uma performance muito boa no quesito inovação é o cenário econômico interno (metas de inflação, câmbio flutuante, superávits primários e cortes das despesas) que tende a prejudicar a efetividade das políticas industriais e de inovação. O país entrou em uma zona de turbulência econômica considerável, com diversos constrangimentos políticos de curto prazo. Para recuperar-se, será necessário aderir a compromissos e resultados de inovação de longo prazo, de forma inteligente e racional (WIPO, 2016).

Apesar disso, o Brasil possui considerável infraestrutura e massa crítica científica e tecnológica, tanto pública quanto privada, para projetos de P\&D. Porém, esses acabam atendendo de forma limitada as demandas dos projetos de inovação dos setores industriais, em função das dificuldades de planejamento, gestão, riscos e falta de recursos humanos dedicados à inovação (EMBRAPII, 2015). O apontamento de tendências tecnológicas relacionadas pelas atividades de produção intelectual e científica pode sugerir caminhos potenciais para a criação de projetos de pesquisa e desenvolvimento.

\section{Metodologia}

Nesta pesquisa as bases de dados para coleta e análise das atividades científicas e intelectuais foram a plataforma Espacenet, do escritório europeu de patentes (EPO), como base de patentes e o Web of Science e Scopus como bases de artigos científicos para a coleta.

Primeiro, a escolha da Espacenet é justificada pela sua abrangência territorial e livre acesso, compreendendo patentes registradas no mundo inteiro. Salienta-se que existem bases diversas de consulta de patentes que são públicas, como o EPO, compreendendo patentes do mundo todo; Instituto Nacional de Propriedade Industrial (INPI), patentes brasileiras; Escritório de Marcas e Patentes dos Estados Unidos (USPTO), base de patentes norteamericanas; Pesquisa de patentes do Google, base de patentes norte-americanas e muitas outras.

A escolha da Scopus e da Web of Science, é factível pois estão entre as melhores bases científicas existentes (Wang; Waltman, 2016; Mongeon; Paul-Hus, 2016; Bar-llan, 2007; Archambault, 2009).

Definidas as bases de pesquisa, houve a definição da String de busca, refinando a mesma para ser realizada em títulos, resumos ou palavras-chave e no intervalo de tempo de 2008 até 2018. A pesquisa retornou termos 
encontrados no título, resumo ou palavras-chave e delimitação temporal de 2008 a 2018. Dado que o conceito em estudo neste artigo é relacionado a smart grids para assim limitar a busca.

Na terceira e última etapa do procedimento metodológico ocorreu a análise dos resultados das duas buscas prévias. Isso possibilitou uma análise descritiva dos dados obtidos separadamente, em cada uma das bases, para auxiliar a compreensão do comportamento dos depósitos anuais no intervalo de tempo definido.

\section{Resultados e discussão}

Nesta sessão são apresentados os resultados obtidos através da busca nas bases de artigos científicos e nas bases de patentes selecionadas. Os dados serão analisados de forma descritiva, possibilitando uma melhor compreensão do comportamento dos depósitos anuais.

\subsection{Análise descritiva: Artigos científicos}

Após a coleta nas plataformas Web of Science e Scopus, os dados foram organizados em planilhas do software Microsoft Excel ${ }^{\circledR}$ por ano. Essa busca retornou um somatório de 8730 artigos científicos identificados com o termo "Smart Grid" em seu título, resumo ou palavras-chave pela Web of Science e 9454 artigos científicos pela Scopus. Após cálculo dos totais de publicações avaliados entre os anos de 2008 e 2018, foram elencados os cinco primeiros países com maiores índices.

A classificação por quantidade de artigos científicos publicados relacionados ao termo buscado pela Web of Science e pela Scopus é apresentada na Tabela 2. Observa-se que, enquanto a Coreia do Sul é apresentada em quinto lugar pela Web of Science, a Scopus apresenta o Reino Unido nessa colocação, com um total de 497 resultados, deixando a Coreia do Sul na sexta posição.

Tabela 1

Total de artigos científicos por país (2008 - 2018).

\begin{tabular}{ccc}
\hline País & Web of Science & Scopus \\
\hline China & 2487 & 2322 \\
EUA & 1580 & 1951 \\
Canadá & 466 & 549 \\
Itália & 360 & 545 \\
Reino Unido & 389 & 497 \\
Coreia do Sul & 322 & 434 \\
\hline
\end{tabular}

A partir da identificação dos cinco países com maior número de publicações registradas, foi possível analisar uma evolução anual de seus depósitos pela Web of Science (Figura 2) e pela Scopus (Figura 3). O comportamento de cada um desses países é apresentado separadamente, podendo-se observar uma possível linearidade no registro anual em países como Estados Unidos, Coréia do Sul, Canadá e Itália em ambos gráficos, e alguns picos de demanda anual em publicações de origem chinesa pela Figura 2, e ainda uma linearidade apresentada no registro de publicações chinesas na Figura 3. 
Figura 2

Evolução de depósitos na Web of Science

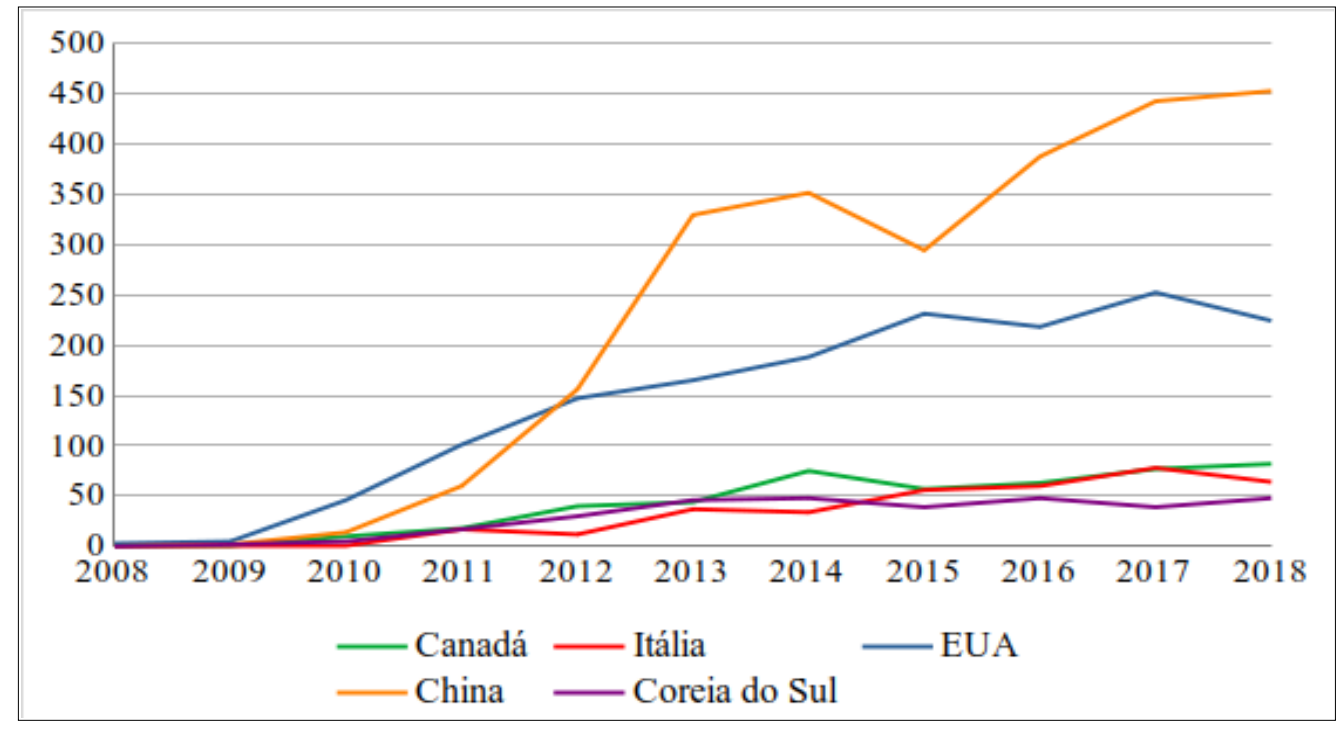

Figura 3

Evolução de depósitos na Scopus

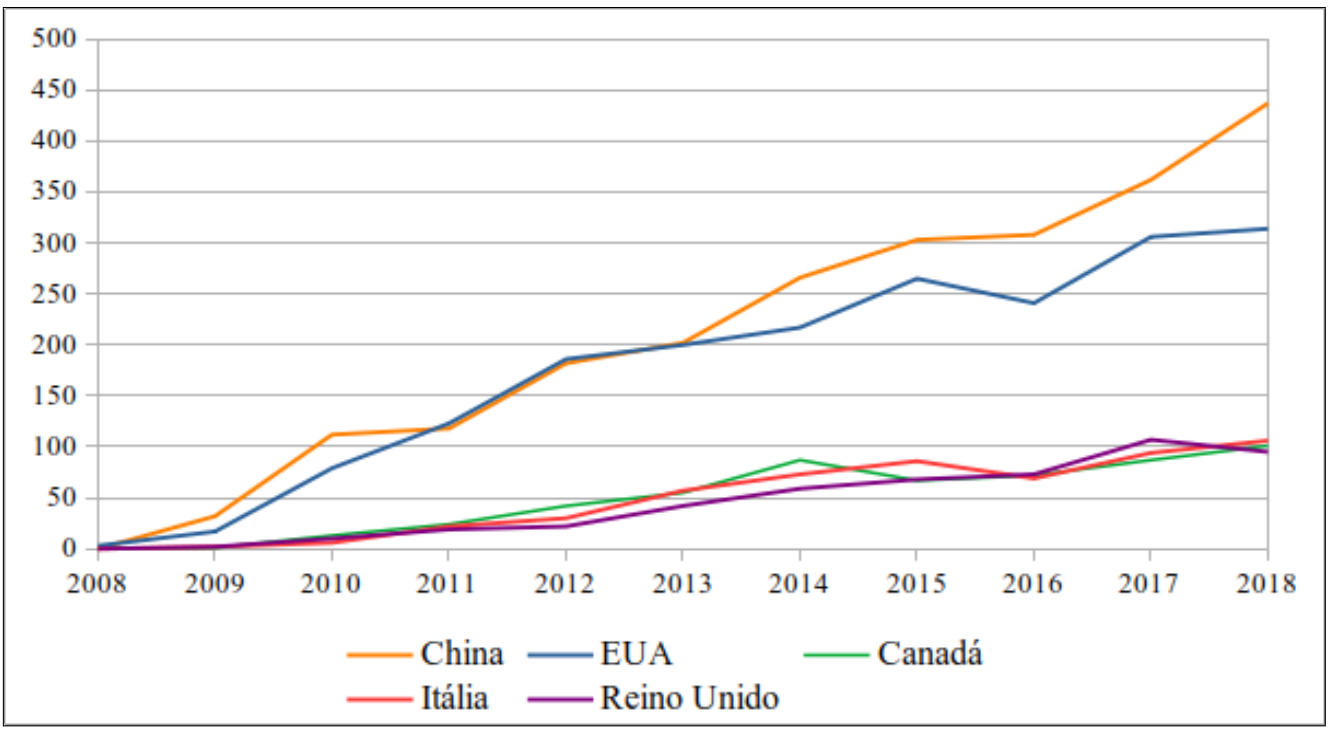

Espera-se que a China continue com maior atividade científica nos próximos anos, pois encontra-se disparado com maiores níveis de publicação em SG do que os outros países. Essa é seguida pelos Estados Unidos. Em relação aos demais países apresentados, as atenções também podem ser voltadas para as atividades acadêmicocientíficas desses países, mas deve-se prestar maior atenção no desenvolvimento de políticas específicas para SGs nos mesmos, visto que são países com destaque nesse mercado e a criação de incentivos pode representar mudanças substanciais nas atividades relacionadas.

\subsection{Análise descritiva: Artigos científicos}

Iniciando o levantamento na Espacenet, foram mantidas as configurações usadas para busca nas bases de artigos científicos. A pesquisa foi definida como uma pesquisa avançada, limitando a procura pelo termo "smart grid", entre aspas, no título ou resumo, limitado pelo ano de busca. 
Assim como na estratificação dos dados da Web of Science e da Scopus, os dados coletados na plataforma Espacenet foram organizados em uma planilha levando em consideração os totais. Esse compêndio retornou uma quantidade de 1049 resultados.

A partir dos totais identificados, foram elencados os países em relação a quantia de publicações encontradas. Foi classificada a China, novamente, como país com maior número de registros de patentes acumulado nesses 10 anos avaliados, com um total de 396 patentes registradas; Coréia do Sul ficou em segundo com 265 registros; Estados Unidos em terceira posição com 149 patentes e, por fim, na quarta e quinta colocação apareceram Japão e Canadá, com 40 e 21 patentes registradas, respectivamente. Além disso, pode ser analisado o comportamento do depósito de patentes de forma geral, cujo crescimento ocorreu de 2009 até 2013, ano que teve seu pico, e tem se apresentado em decrescimento desde então. Cabe observar que 2008 apresentou zero resultados para essa pesquisa.

Após a hierarquização de países com maiores depósitos, foi gerado o gráfico da Figura 4, no qual visualiza-se que China e Coréia do Sul detém números altos e próximos de publicações, até o ano de 2014. Desde então, Coréia do Sul passou a apresentar um decréscimo em sua atividade, impactando fortemente no número de publicações anuais geral. Japão e Canadá apresentaram um comportamento entre si bem semelhante no período, mas muito inferior a China e Coréia do Sul. EUA aparece como intermediário desses dois pares de comportamentos.

Figura 4

Evolução dos depósitos na Espacenet.

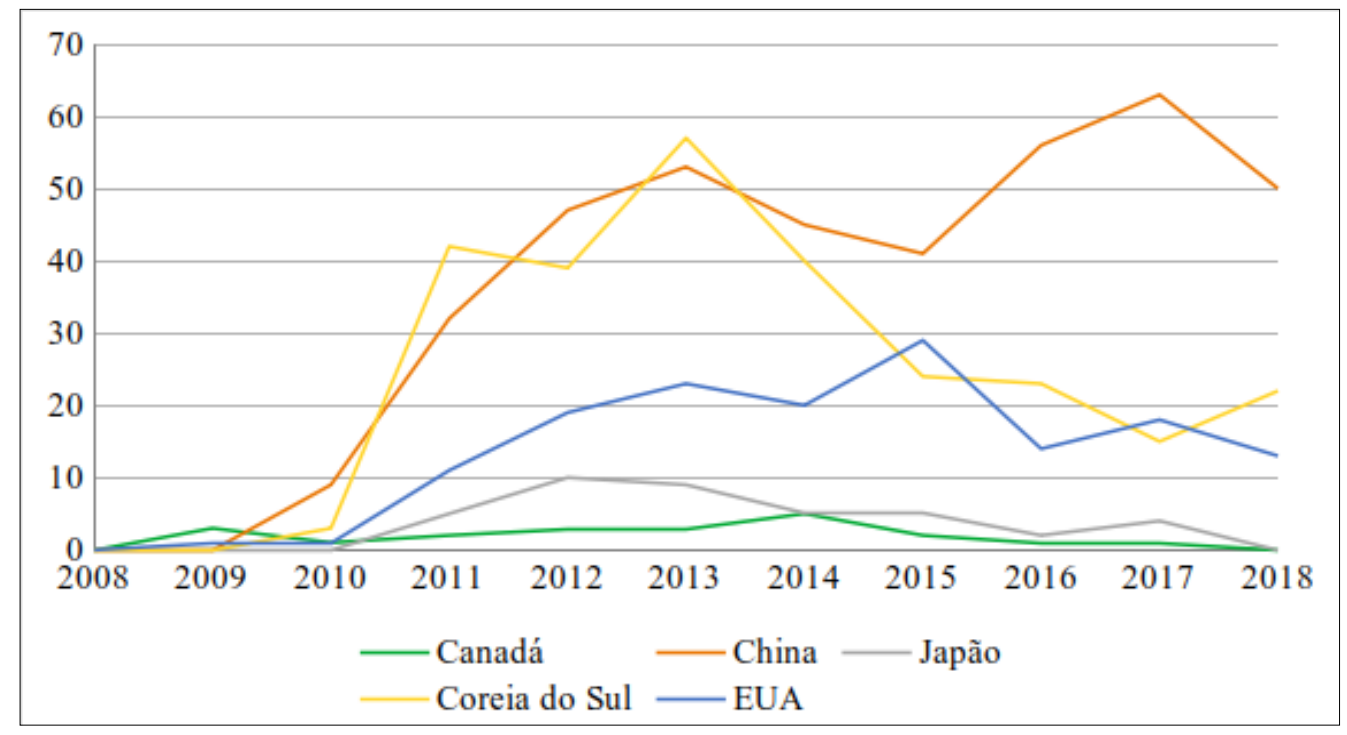

A análise permite inferir que a competição em níveis de patentes está similar ao de publicações científicas, para a área de smart grids, com a diferença de que sai de cena a Itália (50 lugar publicações científicas) e surge o Japão (4ㅇl lugar em patentes). Outro ponto relevante é a aproximação dos níveis de atividade entre Coréia do Sul e EUA a partir de 2015, ocorrido pelo decréscimo acentuado do primeiro país. Muitos outros quesitos despontariam como passíveis de discussão, e juntamente a exploração e validação das análises superficiais já realizadas neste trabalho, tornam-se produto para um segundo estudo.

\section{Conclusão}

O objetivo principal deste trabalho foi de apresentar tendências capazes de auxiliar na identificação de cenários para smart grids do ponto de vista científico e intelectual. Para o Brasil melhorar sua atividade nos itens 
pesquisados neste trabalho é possível dar mais ênfase aos aspectos resultantes, utilizando de maneira estratégica o que as melhores práticas (quem e o que) estão fazendo.

Da análise de produção científica, conclui-se que a China tende a continuar com maior atividade nos próximos anos, pois encontra-se disparado com maiores níveis de publicação em smart grids do que outros países e em linha ascendente. Em relação aos países Canadá, Estados Unidos e Coréia do Sul, as atenções também podem ser voltadas para as atividades acadêmico-científicas individuais desses países. No entanto, é necessário dar atenção no desenvolvimento de políticas específicas, visto que são países com destaque e a criação de incentivos pode representar mudanças substanciais nas atividades relacionadas.

Quanto a análise da produção intelectual (patentes), visualiza-se que a curva que representa a atividade de registro de patentes da China tem um comportamento similar a uma curva "S", demonstrando nível relativamente alto de variação, e a tendência sugere estar ligeiramente decrescendo. A Coréia do Sul esteve à frente da China em meados de 2013 e depois mostrou queda. Porém, em 2018 a Coréia do Sul apresenta sinal de retorno do crescimento. EUA também é reforçado como país de atenção para próximos anos no quesito.

As limitações desta pesquisa residem nos seus aspectos metodológicos das bases de dados utilizadas e nas inferências realizadas. Um dos pontos em comum às bases de dados científica e de patentes é o conteúdo que ambas disponibilizam. Apesar de amplos, podem deixar dados relevantes de fora. Também, a análise quantitativa realizada considera apenas o número de registros em cada país, desconsiderando possíveis variáveis demográficas que podem ser um fator importante relacionado a totalidade de publicações em cada país, interferindo diretamente ao seu posicionamento no ranking.

Como sugestões de trabalhos futuros fica a ideia da expansão das bases utilizadas, tanto de forma isolada como complementar às já utilizadas. Outra sugestão é a de criação de um framework para reprodutibilidade da sistemática desta pesquisa, o que pode permitir a criação de dados para validação do processo e permitir acoplamento de módulos que compreendam outros indicadores das regiões analisadas, potencializando a legitimação das tendências apontadas.

\section{Agradecimentos}

Este trabalho foi apoiado pelo Conselho Nacional de Desenvolvimento Científico e Tecnológico (CNPq) e Fundação de Amparo à Pesquisa do Estado do Rio Grande do Sul (FAPERGS) pelo apoio a esta pesquisa.

\section{Referências bibliográficas}

Alves, V. T., Mairesse-Siluk, J. C., Neuenfeldt-Júnior, A. L., Soliman, M., \& Dalla-Nora, L. D. (2015). Performance assessment of internal logistics for service companies. Revista Facultad de Ingeniería Universidad de Antioquia, 74(Jan./Mar.), 188-199.

Archambault, É., Campbell, D., Gingras, Y., \& Larivière, V. (2009). Comparing bibliometric statistics obtained from the Web of Science and Scopus. Journal of the American Society for Information Science and Technology, 60(7), 1320-1326.

Bar-llan, J., Levene, M., \& Lin, A. (2007). Some measures for comparing citation databases. Journal of Informetrics, 1(1), 26-34.

Battistella, C. (2014). The organisation of Corporate Foresight: A multiple case study in the telecommunication industry. Technological Forecasting and Social Change, 87(September), p. 60-79. 
Caruso, L. A., \& Tigre, P. B. (2004). Modelo Senai de Prospecção: documento metodológico. Montevidéo: Cinterfor/OIT.

Carvalho, M., \& Delgado, D. (2017). Potential of photovoltaic solar energy to reduce the carbon footprint of the Brazilian electricity matrix. Revista Latino-Americana em Avaliação do Ciclo de Vida, 1(1), 64-85.

Cavallo, E., Eichengreen, B. \& Panizza, U. (2018). Can countries rely on foreign saving for investment and economic development?. Review of World Economics, 154(2), 277-306.

CGEE (2014). Parcerias estratégicas. Recuperado no dia 17 de agosto de 2019, de http://seer.cgee.org.br/index.php/ parcerias_estrategicas/issu/view/76.

Chan, L. \& Daim, T. (2012). Exploring the impact of technology foresight studies on innovation: Case of BRIC countries. Futures, 44(6), 618-630.

Costa, R., Siluk, J., Neuenfeldt Júnior, A., Soliman, M., \& Nara, E. (2015). A gestão da competitividade industrial por meio da aplicação dos métodos UP e multicritério no setor frigorífico de bovinos. Ingeniare. Revista chilena de ingeniería, 23(3), 383-394.

Dağ, B., Boynueğri, A. R., Ateş, Y., Karakaş, A., Nadar, A., \& Uzunoğlu, M. (2016). Static modeling of microgrids for load flow and fault analysis. IEEE Transactions on Power Systems, 32(3), 1990-2000.

EMBRAPII (2014). Sistema de Excelência Operacional EMBRAPII. Recuperado no dia 07 de agosto de 2018, de http://embrapii.org.br/wpcontent/uploads/2014/12/lo_embrapii_sistemasexcelenciaoperacional.pdf.

EMBRAPII (2015). Plano Diretor 2014-2019. Recuperado no dia 07 de agosto de 2018, de http://embrapii.org.br/wpcontent/uploads/2014/09/plano-diretor-embrapii.pdf.

ESPACENET (2019). Espacenet: free access to over 110 million patent documents. European Patent Office. Recuperado no dia 10 de maio de 2019, de https://worldwide.espacenet.com/.

Falbo, R. D. A., Fernandes, T. L., Lima, L. A., Tenório, L. X. Da S., Silva, M. L. Da, Ghesti, G. F., \& Mayerhoff, Z. D. V. L. (2015). Uma Análise Sobre os Estudos de Prospecção Tecnológica. Cadernos de Prospecção, 9(4), 7-9.

Farhangi, H. (2010). The path of the smart grid. IEEE Power and Energy Magazine, 8(1), 18-28.

Garlet, T. B., Ribeiro, J. L. D., de Souza Savian, F., \& Siluk, J. C. M. (2019). Paths and barriers to the diffusion of distributed generation of photovoltaic energy in southern Brazil. Renewable and Sustainable Energy Reviews, 111(September), 157-169.

Júnior, A. L. N., Siluk, J. C. M., Soliman, M., \& Paris, S. D. (2018). Modelling for performance measurement of bus rapid transit systems in Brazil. International Journal of Logistics Systems and Management, 30(3), 283-297.

Kubli, M. (2018). Squaring the sunny circle? On balancing distributive justice of power grid costs and incentives for solar prosumers. Energy policy, 114(March), 173-188.

Mayerhoff, Z. D. V. L. (2009). Uma análise sobre os estudos de prospecção tecnológica. Cadernos de Prospeç̧ão, 1(1), 7-9.

Mazzucato, M., \& Penna, C. (2016). The Brazilian innovation system: a mission-oriented policy proposal. Recuperado no dia 08 de maio de 2019, de https://www.cgee.org.br/ documents/10195/1774546/Sistema_Brasileiro_de_Inovacao-Mazzucato_Penna-Sumario_Executivo.pdf. 
Ministério de Minas e Energia - MME (2016). Energia Solar no Brasil e Mundo. Recuperado no dia 18 de dezembro de 2019, de http://www.mme.gov.br/documents/10584/3580498/17+-+Energia+Solar++Brasil+e+Mundo+-+ano+ref.+2015+\%28PDF\%29/4b03ff2d-1452-4476-907d-d9301226d26c?version=1.3.

Mongeon, P., \& Paul-Hus, A. (2016). The journal coverage of Web of Science and Scopus: a comparative analysis. Scientometrics, 106(1), 213-228.

Nascimento, F. M. D., Neuenfeldt Júnior, A., De Paris, S., Stieler, G., \& De Moreira, L. A. A. (2019). A mensuração de desempenho organizacional para a abertura de uma distribuidora de bebidas. Revista Espacios, 40(35), 6.

Neuenfeldt Junior, A. L., Siluk, J. C. M., Soliman, M., Nara, E. O. B., \& Kipper, L. M. (2015). Hierarchy the sectorial performance indicators for Brazilian franchises. Business Process Management Journal, 21(1), 190-204.

Rediske, G., Siluk, J. C. M., Gastaldo, N. G., Rigo, P. D., \& Rosa, C. B. (2018) Determinant factors in site selection for photovoltaic projects: a systematic review, Int J Energy Res, 43, 1689-1701.

Rosa, C. B., Siluk, J. C. M., \& Michels, L. (2016). Proposal of the instrument for mea-suring innovation in the generation photovoltaics. IEEE Lat Am Trans, 14, 4534-4539.

Sadoogi, B., \& Najafzadeh, M. R. (2016). Development of marketing using systemic process of competitive intelligence and foresight - future studies. European Journal of Physical Education and Sport Science, $1(4), 1$.

Salisbury, L. (2009). Web of Science and Scopus: A comparative review of content and searching capabilities. The Charleston Advisor, 11(1), 5-18.

Sampaio, P. G. V., \& González, M. O. A. (2017). Photovoltaic solar energy: Conceptual framework. Renewable and Sustainable Energy Reviews, 74, 590-601.

Scarano, T. F., Siluk, J. C. M., Nara, E. O. B., Júnior, A. L. N., \& Da Fontoura, F. B. B. (2014). Diagnóstico do desempenho organizacional em empresas do setor metal mecânico. Revista Espacios, 35 (3), 18.

Schandl, H., Hatfield-Dodds, S., Wiedmann, T., Geschke, A., Cai, Y., West, J., Newth, D., Baynes, T., Lenzen, M., \& Owen, A. (2016). Decoupling global environmental pressure and economic growth: scenarios for energy use, materials use and carbon emissions. Journal of Cleaner Production, 132(20), 45-56.

Siano, P. (2014). Demand response and smart grids -A survey. Renewable and Sustainable Energy Reviews, 30, 461-478.

Siluk, J. C. M., Kipper, L. M., Nara, E. O. B., Neuenfeldt Junior, A. L., Dal Forno, A. J., Soliman, M., \& Chaves, D. M. D. S. (2017). A performance measurement decision support system method applied for technologybased firms' suppliers. Journal of Decision Systems, 26(1), 93-109.

Silva, A. M., \& Vieira, R. M. F. (2016). Energia eólica: conceitos e características basilares para uma possível suplementação da matriz energética brasileira. Revista Direito Ambiental e Sociedade, 6(2), 1.

Wang, Q. \& Waltman, L. (2016). Large-scale analysis of the accuracy of the journal classification systems of Web of Science and Scopus. Journal of Informetrics, 10(2), 347-364.

Web of Science (2019). Coleção Principal, Clarivate Analytics. Recuperado no dia 10 de maio de 2019, de https://www.webofknowledge.com/. 
Wipo (2011) The Global Innovation Index 2011: Accelerating growth and development. Recuperado no dia 15 de dezembro de 2019, de http://www.wipo.int/publications/en/details.jsp?id=274.

Wipo (2012) The Global Innovation Index 2012: Stronger innovation linkages for global growth. Recuperado no dia 15 de dezembro de 2019, de http://www.wipo.int/publications/en/details.jsp?id=247.

Wipo (2013) The Global Innovation Index 2013: The local dynamics of innovation. Recuperado no dia 15 de dezembro de 2019, de http://www.wipo.int/edocs/pubdocs/en/economics/gii/gii_2013.pdf.

Wipo (2014) The Global Innovation Index 2014: The human factor in innovation. Recuperado no dia 15 de dezembro de 2019, de http://www. wipo.int/publications/en/details.jsp?id=3254.

Wipo (2015) The Global Innovation Index 2015: Effective innovation policies for development. Recuperado no dia 15 de dezembro de 2019, de http://www.wipo.int/edocs/pubdocs/en/wipo_gii_2015.pdf.

Wipo (2016) The Global Innovation Index 2016: Winning with global innovation. Recuperado no dia 15 de dezembro de 2019, de http://www.wipo.int/econ_stat/en/economics/gii/.

Wipo (2017) The Global Innovation Index 2017: Innovation nourishing the world. Recuperado no dia 15 de dezembro de 2019, de https://www.globalinnovation index.org/userfiles/file/reportpdf/GII\%202017\%20Portuguese\%20translation_WEB.pdf.

Wipo (2018) The Global Innovation Index 2018: Energizing the world with innovation. Recuperado no dia 15 de dezembro de 2018, de https://www.global innovationindex.org/gii-2018-report\#.

Esta obra está bajo una Licencia Creative Commons Attribución-NoCommercial 4.0 International

\section{(c) EY-NO}

